\title{
Combined spinal-epidural anesthesia in a mild thrombocytopenic patient with antiphospholipid antibody syndrome
}

\author{
Gahyun Kim, Hyunyee Sim, Jaeyoung Yang, Jong-Hwan Lee, and Duck Hwan Choi \\ Department of Anesthesiology and Pain Medicine, Samsung Medical Center, Sungkyunkwan University School of Medicine, Seoul, \\ Korea
}

A hypercoagulable state in antiphospholipid antibody syndrome (APS) may cause many obstetric complications during the pregnancy and postpartum. Therefore, a pregnant woman with APS should be treated with aspirin alone or combined with either unfractionated heparin or low molecular weight heparin [1]. With antithrombotic prophylaxis, thrombocytopenia can often occur in a pregnant woman with APS, and those conditions can cause confusions when conducting regional anesthesia for cesarean section.

A 30-year-old woman (height of $160.4 \mathrm{~cm}$ and body weight of $73.7 \mathrm{~kg}$ ) was admitted for the elective repeat cesarean section at gestational age of 39 weeks. She had an obstetric history of 5 recurrent early pregnancy losses, and only one live birth was given 3 years ago with cesarean section under empirical aspirin therapy. The cause of the previous cesarean section was expected to be the low birth weight, and general anesthesia was performed due to the persistent thrombocytopenia unresponsive to platelet transfusions. She was finally diagnosed with APS through laboratory test one year ago at the time of her last abortion. The cesarean section was performed under general anesthesia due to the low platelet count, and further evaluation was not conducted. She took aspirin $100 \mathrm{mg}$ /day and hydroxychlorquine $200 \mathrm{mg}$ twice a day according to the recommendation of a rheumatologist. After confirming her pregnancy at 6 weeks of gestational age, she started to receive enoxaparin $40 \mathrm{mg}$ subcutaneously once daily throughout the pregnancy. There was no significant event during her pregnancy.

On the admission for elective repeat cesarean section, her platelet count was $85,000 / \mu \mathrm{l}$ and there was no significant abnormality in the coagulation tests and liver function profiles. Thrombocytopenia had been observed since third trimester and was maintained stable without bleeding tendency such as bruises and petechiae. After consulting with the obstetrician, we decided to perform combined spinal-epidural anesthesia for her cesarean section.

She was administered aspirin until the morning of the surgery, and she was sent to the operating room 10 hours after the last subcutaneous injection of enoxaparin $40 \mathrm{mg}$. With the patient in the right lateral position, a 19-gauge epidural catheter (FlexTip plus ${ }^{\circledR}$; Arrow International, Inc., Reading, PA, USA) was inserted via a 17-gauge Tuohy needle at the L2-3 intervertebral space, using a midline approach with the loss-of-resistanceto-air technique and fixed at $10 \mathrm{~cm}$ to the skin. Then, spinal tapping was done at the L3-4 interspace with 25-gauge Whitacre needle and $6 \mathrm{mg}$ of $0.5 \%$ hyperbaric bupivacaine, and $20 \mu \mathrm{g}$ of fentanyl were administered intrathecally. An epidural injection of $10 \mathrm{ml}$ of $0.25 \%$ levobupivacaine followed thereafter. The sensory block to the T4 level was achieved with additional injection of $10 \mathrm{ml}$ of $2 \%$ lidocaine. Her vital signs were maintained stable during the operation with just one dose of phenylephrine (100

Corresponding author: Duck Hwan Choi, M.D., Ph.D., Department of Anesthesiology and Pain Medicine, Samsung Medical Center, Sungkyunkwan University School of Medicine, 81, Irwon-ro, Gangnam-gu, Seoul 135-710, Korea. Tel: 82-2-3410-0369, Fax: 82-2-3410-0361, E-mail: duckhwanc@gmail.com

(c) This is an open-access article distributed under the terms of the Creative Commons Attribution Non-Commercial License (http:// creativecommons.org/licenses/by-nc/3.0/), which permits unrestricted non-commercial use, distribution, and reproduction in any medium, provided the original work is properly cited. 
$\mu \mathrm{g}$ ). A $3.52 \mathrm{~kg}$ healthy male infant was delivered (APGAR score of 9 at 1 and $5 \mathrm{~min}$ ). Total anesthetic time was $101 \mathrm{~min}$, and the cesarean section was completed without any event. T6 sensory block was identified just upon the arrival to the postanesthesia care unit. Then, a patient-controlled epidural analgesia was started with $0.11 \%$ ropivacaine and $3.7 \mu \mathrm{g} / \mathrm{ml}$ fentanyl through the epidural catheter, for postoperative pain control (bolus-lockout time-basal, $2 \mathrm{ml}$ - $15 \mathrm{~min}-4 \mathrm{ml}$ ). Enoxaparin was re-started in the absence of postpartum bleeding on the next morning which was 24 hours after the operation and was scheduled to be used for up to 6 weeks postoperative. The patient didn't have any symptoms or signs of venous thromboembolism during peripartum period. The epidural catheter was removed 2 hours before the regular enoxaparin injection on postoperative day 2 . She was discharged on postoperative day 4 without any neurologic complication.

Compared with normal pregnant women, the pregnant women with APS are approximately 15 times more likely to develop thromboembolism, and the risk is increased by 24 -fold in the cases of thrombosis history [2]. Deep vein thrombosis or pulmonary embolism was seen at a lower incidence in patients under regional anesthesia than general anesthesia [3], and satisfactory postoperative pain control with patient-controlled epidural analgesia would allow early ambulation to decrease the risk of thrombosis. Based on the American Society of Regional Anesthesia and Pain Medicine Evidence-Based Guidelines, even though prophylactic dose of $40 \mathrm{mg}$ of subcutaneous enoxaparin is taken once daily, neuraxial block could be executed 10-12 hours following the last dose. Based on the guideline, the epidural catheter was removed 2 hours before the next dose. Most of the spinal hematoma in patients receiving enoxaparin was reported when prophylactic dose of enoxaparin was 30-40 mg twice daily or higher [4].

Recently, regional anesthesia has been performed safely in pregnant patients with platelet counts between $50-79 \times 10^{3} / \mu \mathrm{l}$. Normal hemostasis can be maintained until the platelet count decreases to $54 \times 10^{3} / \mu \mathrm{l}$ [5]. Stable mild thrombocytopenia should not be a contraindication for regional anesthesia, as long as there is no bleeding diathesis, significant prolongation of prothrombin time or APTT, decreasing tendency of platelet counts, or association with eclampsia. In this case, we carefully monitored the possible clinical symptoms of spinal hematoma, because a continuous epidural catheter was adopted during postpartum period even though the patient still had bleeding tendency. We didn't consider the thromboelastogram or the platelet function analyzer; however, it would have been more secure if the platelet function was evaluated [5]. A continuous neuraxial block could mask the symptoms of motor or sensory changes from spinal hematoma. Therefore, close observation is essential for early detection of spinal hematoma.

APS with thrombosis in pregnancy requires higher dose of anticoagulation (enoxaparin $1 \mathrm{mg} / \mathrm{kg}$ ). In such case, general anesthesia is much safer to avoid spinal hematoma. General anesthesia might also be favorable in emergent situation such as fetal distress due to placental infarction in a patient with APS, because anesthetic induction time is shorter than the regional anesthesia.

Regional anesthesia would also be considered as a safe anesthetic option in a patient with APS syndrome, antithrombotic prophylaxis, and mild thrombocytopenia. Anesthesiologists should consider the use of anticoagulants and practical coagulation state to choose anesthetic technique for cesarean section in a patient with APS, by weighing the benefits and the risks between general or regional anesthesia.

\section{References}

1. Di Prima FA, Valenti O, Hyseni E, Giorgio E, Faraci M, Renda E, et al. Antiphospholipid Syndrome during pregnancy: the state of the art. J Prenat Med 2011; 5: 41-53.

2. James AH, Jamison MG, Brancazio LR, Myers ER. Venous thromboembolism during pregnancy and the postpartum period: incidence, risk factors, and mortality. Am J Obstet Gynecol 2006; 194: 1311-5.

3. Rodgers A, Walker N, Schug S, McKee A, Kehlet H, van Zundert A, et al. Reduction of postoperative mortality and morbidity with epidural or spinal anaesthesia: results from overview of randomised trials. BMJ 2000; 321: 1493.

4. Horlocker TT, Wedel DJ, Rowlingson JC, Enneking FK, Kopp SL, Benzon HT, et al. Regional anesthesia in the patient receiving antithrombotic or thrombolytic therapy: American Society of Regional Anesthesia and Pain Medicine Evidence-Based Guidelines (Third Edition). Reg Anesth Pain Med 2010; 35: 64-101.

5. Tanaka M, Balki M, McLeod A, Carvalho JC. Regional anesthesia and non-preeclamptic thrombocytopenia: time to re-think the safe platelet count. Rev Bras Anestesiol 2009; 59: 142-53. 